\title{
What is to be learnt? Critical aspects of elementary arithmetic skills
}

\author{
Camilla Björklund $^{1}$ (D) $\cdot$ Ference Marton ${ }^{2} \cdot$ Angelika Kullberg $^{2}$
}

Accepted: 22 February 2021/ Published online: 20 March 2021

(C) The Author(s) 2021

\begin{abstract}
In this paper, we present a way of describing variation in young children's learning of elementary arithmetic within the number range 1-10. Our aim is to reveal what is to be learnt and how it might be learnt by means of discerning particular aspects of numbers. The Variation theory of learning informs the analysis of 2184 observations of 4- to 7year-olds solving arithmetic tasks, placing the focus on what constitutes the ways of experiencing numbers that were observed among these children. The aspects found to be necessary to discern in order to develop powerful arithmetic skills were as follows: modes of number representations, ordinality, cardinality, and part-whole relation (the latter has four subcategories: differentiating parts and whole, decomposing numbers, commutativity, and inverse relationship between addition and subtraction). In the paper, we discuss particularly how the discernment of the aspects opens up for more powerful ways of perceiving numbers. Our way of describing arithmetic skills, in terms of discerned aspects of numbers, makes it possible to explain why children cannot use certain strategies and how they learn to solve tasks they could not previously solve, which has significant implications for the teaching of elementary arithmetic.
\end{abstract}

Keywords Arithmetic skills · Critical aspects · Preschool children · Variation theory

Camilla Björklund

camilla.bjorklund@ped.gu.se

Ference Marton

ference.marton@ped.gu.se

Angelika Kullberg

angelika.kullberg@ped.gu.se

1 Department of Education, Communication and Learning, University of Gothenburg, Box 300, 405

30 Gothenburg, Sweden

2 Department of Pedagogical, Curricular and Professional Studies, University of Gothenburg,

Gothenburg, Sweden 


\section{What is to be learnt?}

When a group of learners embark upon a new curricular unit, it seems reasonable to expect educational objectives to address the question "What is to be learnt?". At best, however, we might find out what the chosen group of students are expected to be able to do upon their completion of the curricular unit in question. This follows from the fact that the educational objectives are presumably the same for all students of the same curricular unit, while we can safely assume that the students differ in what they need to learn to achieve the objectives of the curricular unit. If their prerequisites differ, they need to learn different things to achieve the same goal. In this paper, we present a way of describing variation in learning, and apply this to the study of young children's learning of elementary arithmetic within the number range 1-10. Our aim is to reveal what is to be learnt as well as how it might be learnt. To fulfil this aim, we observed 103 children's attempts to solve eight arithmetic tasks on three occasions and analysed what constitutes their ways of experiencing numbers. As outlined above, they differ in their ways of understanding and using numbers, and thereby have different prerequisites for solving the tasks.

Earlier research on arithmetic skills among young students has indeed presented thorough observations of what children do when encountering arithmetic tasks, resulting in descriptions of trajectories in the development of arithmetic skills (e.g., Fuson, 1982, 1992). Other scholars, such as Steffe (2004) have made efforts to describe the processes of constructing schemes that open the ability to solve tasks that are more advanced. However, this previous research has not to a sufficient extent shown what is the actual potential for arithmetic learning that different strategies in arithmetic problem solving may have, which presumably has to do with different foci and aims of the research. The constructivist view, which Steffe is a representative of, interprets arithmetic knowledge as constructed through mental activities and sees development in light of more complex forms of thinking. However, when taking a phenomenological standpoint, knowledge is constituted through an internal relationship between the child and the object of learning. Researching development is then aiming to explore the world as it is experienced by the child. The difference in these theoretical views thus refers to different interpretations of what it takes to develop arithmetic skills (Marton \& Neuman, 1990). One example of children's ways of solving arithmetic tasks that both Fuson and Steffe mention is so-called double counting ${ }^{1}$. Fuson has observed this strategy used among young children as one of many possible strategies in arithmetic problem solving. Steffe interprets this strategy to be an expression of the child having constructed a "tacitly nested number sequence" (Steffe, 2004, p. 236), which may bridge to strategic reasoning in addition and subtraction. In the case of double counting strategy, we argue based on empirical studies (Neuman, 1987) and particularly the theoretical framework Variation theory (with origins in phenomenological ontology), that double counting does not bridge to more advanced understanding since the strategy applied is an expression of a certain way of perceiving numbers, in this case as parallel number sequences (see also Björklund, 2021). Strategic reasoning in problem solving is possible only if the problem solver perceives numbers in ways that allow such reasoning. The act of double counting does not induce such an awareness of the number relations that is

\footnotetext{
${ }^{1}$ Double counting is a way of keeping track in a counting task where the sequence of counting words are treated as entities to be counted. When solving e.g., $10-6=$ the child keeps track of how many units are taken away and how many are left, at the same time, usually by raising one finger for each counted counting word and then counting the raised fingers, or by indexing with number words: " 10,9 (1 taken away), 8(2).. 4(6)", thus the numbers are double counted.
} 
necessary for developing strategic arithmetic reasoning. Our approach may thus contribute to the field of research by taking a pedagogical perspective on the learning of arithmetic skills founded in Variation theory of learning, in our quest to describe observed variations in learning, and in particular to conceptualise how the learning of numbers' meaning proceed in terms of what is to be learnt and how this might be learnt.

In our approach to research on learning and teaching elementary arithmetic in the number range $1-10$, we assume, based on principles from Variation theory of learning that there are certain aspects of the first ten numbers that learners have to learn to discern in order to solve certain types of arithmetic tasks. The children, for instance, have to learn to discern the cardinality — or "manyness" - of two numbers in order to add them, thus forming a third cardinality, the sum of the two cardinalities. Now, such aspects that the learner has to discern but has not yet discerned are exactly "what is to be learnt". This is why we call them "critical aspects" (Marton, 2015, p. 24). While the necessity to learn the cardinal meaning of numbers has been proposed in many studies before ours (e.g., Gelman \& Gallistel, 1978; Sarnecka et al., 2018; Wynn, 1992), how such understanding develops, and how this attribute of numbers relates to other ones, is less well known.

A key feature of the theoretical framework we apply in our inquiry is that the meaning of an attribute appears mainly in comparison with other attributes of the same kind. For instance, the meaning of "green" appears in comparison with other colours, the meaning of "natural numbers" appears through comparison with other kinds of numbers, and the meaning of a certain natural number appears in comparison with other natural numbers (see Marton, 2015). Thus, we must encounter at least two different numbers, for instance, one represented by one thing and the other by two things of the same kind. At the same time, it is through the comparisons that we can see what different (but comparable) attributes (e.g., natural numbers) have in common, namely that they can be seen in relation to each other as values in a certain dimension of variation. "Aspect" and "dimension of variation" are synonyms in the present context, and so are "attribute" and "value". Single attributes can then be seen as component parts of systems of attributes.

This is also true in elementary arithmetic. In this paper, we will show that discerning critical aspects of numbers, in terms of dimensions of variation and distinguishing between varying attributes in these dimensions, is key to understanding children's developing arithmetic skills. Differences between learners can be seen in terms of the aspects of the object of learning that are - or are not - discerned by them. Another way of saying this is that differences in learners' success in solving tasks are found in how they perceive these tasks (i.e., in terms of what aspects of the tasks that are seen by them). This further leads to a theoretically argued (Marton \& Booth, 1997; Marton, 2015) and empirically shown way of how teachers may create necessary conditions for learning (see Björklund et al., 2021; Marton \& Tsui, 2004). One example from a larger research project ${ }^{2}$ is the positive outcomes of an intervention study, in which children who were afforded to experience contrasting attributes of numbers learnt to use more powerful ways for solving arithmetic problems than a

\footnotetext{
${ }^{2}$ The project we here refer to is FASETT, conducted during 2015-2018, of which the study in this particular article is one part. The project's main aim was to investigate the foundational aspects of arithmetic skills development and how young children's ability to discern these aspects could be developed using a pedagogy based on specific empirical and theoretical insights.
} 
comparison group (Kullberg et al., 2020). It is our goal with the present study to further explore how these positive outcomes can be explained, on a theoretical basis.

\subsection{Five conjectures of the learning of elementary arithmetic skills}

In accordance with the introduction above, we will advance five conjectures ${ }^{3}$ derived from the application of the Variation theory of learning (Marton, 2015) and Neuman's (1987) theory of the origin of arithmetic skills. These conjectures reflect some of the main principles of the theory that we are using in our study. The conjectures originate mainly from the research referred to in the present paper. By means of our line of reasoning, we intend to relate previous and novel findings to strengthen the validity of the conjectures and support our conclusions as far as the learning of elementary arithmetic is concerned.

First, differences in young children's ways of handling arithmetic tasks derive from differences in their ways of perceiving ${ }^{4}$ these tasks.

Second, differences in young children's ways of perceiving arithmetic tasks derive from which aspects of these tasks they simultaneously discern and focus on.

Third, an aspect of an arithmetic task is a dimension in which commensurable (i.e., comparable) attributes may vary in the learners' awareness (e.g., the cardinality of numbers is an attribute that varies in the dimension of cardinality).

Fourth, learners may fail to solve an arithmetic task because they do not discern one or several necessary aspects of it. Such aspects are said to be "critical" to discern, i.e., necessary for the learner to solve the task but not yet attained. This means that children may discern some necessary aspects of the object of learning but not others (they are to be learnt). Hence, their often seemingly erroneous understanding of the object of learning (that which is to be learnt) is not so much erroneous as incomplete.

Fifth, teachers can help learners to discern critical aspects by bringing about patterns of variation and invariance within and between tasks that are instances of the object of learning. In order to experience such patterns of difference and sameness, they must be present to the child. This is how the teacher might create the conditions necessary for learning — necessary, though not necessarily sufficient.

The outlined conjectures derive from multiple studies of learners' encounters with - and handling of - tasks in different knowledge areas, constituting some of the main principles of the Variation theory of learning (Marton \& Booth, 1997; Marton \& Tsui, 2004). In this paper, we apply these conjectures to the inquiry into the development of children's arithmetic skills. Marton and Neuman (1989) argued that being able to experience - or to perceive - the numbers $1-10$ and the relations between them is a necessary condition for the mastery of elementary arithmetic. Neuman's (1987) empirical findings further show that difficulties in developing arithmetic skills may be due to the learner not simultaneously experiencing the numbers' ordinality, cardinality and relations between them. Moreover, the Variation theory of learning implies that discerning these necessary aspects of numbers is likely to occur when relevant tasks are juxtaposed in relevant ways. Our conjectures further imply that learning is a

\footnotetext{
${ }^{3}$ Conjectures here mean expectations or "propositions without proof".

${ }^{4}$ In accordance with the Variation theory of learning, the expressions "perceive", "to see" (sensuously or metaphorically) and "to experience" are used as synonyms (Marton, 1981).
} 
change in ways of seeing (or perceiving). If a child is only able to see some aspects but not others, this may limit what they are able to do with numbers (thus limiting their arithmetic skills). In this sense, to experience patterns of variation is a kind of "liberation", because when a new dimension of variation is opened and children experience numbers in a new way, they are also able to do things with numbers that they were not able to do before.

In line with the conjectures, learning to experience numbers in certain ways does not happen through the repetition of acts (or performing arithmetic procedures). More than 80 years ago, Brownell stated that "If one is to be successful in quantitative thinking one needs a fund of meanings, not a myriad of 'automatic responses"' (1935, p. 10). He further argued that the repetition of arithmetic facts will not lead to understanding. This has been confirmed and theoretically founded in more recent investigations of learning processes: it is the variation in what learners perceive while repeating an act that enables them to develop new and powerful ways of understanding the content in question (Marton, 2015; Marton \& Tsui, 2004; Marton et al., 2005). In this sense, powerful ways of solving tasks do not only mean to produce correct answers. Rather, the way of approaching a task with flexibility in strategy choice and handling number relations that supports sustainable and developable ways of thinking about arithmetic tasks, constitutes "powerful ways of seeing". Thus, it seems appropriate to assume that learning how to solve arithmetic tasks has to do with how children experience numbers in the arithmetic tasks that enable the child to handle them in powerful ways or limit them in doing so. We present arguments for this claim by studying what aspects of numbers children experience while enacting different strategies to solve the tasks. This way of interpreting arithmetic learning thus provides a framework for understanding and describing why development occurs and what teaching elementary arithmetic should attend to.

\section{Research on arithmetic skills development}

The development of arithmetic skills is perhaps the most discussed field in mathematics education research but is also described from quite different perspectives with different implications for mathematics teaching. Since our intention is to contribute with a complementary way of describing arithmetic development and what is to be learnt, we will here briefly discuss the field of knowledge as it is typically posited in the large body of literature.

Fuson $(1982,1992)$ has provided the field of knowledge extensive descriptions of children's strategies in arithmetic problem solving and created a trajectory of more and more advanced strategies and explanations of what the child has to be able to do before advancing on the trajectory. The observed strategies often start with "counting all" and then advance to "counting on", and in subtraction tasks "counting down" (Fuson, 1992). These strategies are based on the child's way of solving the task (first counting and then determining the quantity of the set as "count-to-cardinal" induces the "counting all" strategy while "cardinal-to-count" where the set is first approximated and then counted is assumed a prerequisite approach for the "counting on" strategy). Her observations have been confirmed in many studies and complemented by cognitive theory, indicating that there are certain processes enabling the child to eventually construct or "find" arithmetical meaning and strategies (see Steffe, 2004; Steffe et al., 1988). In this research, schemes are important for explaining children's mathematical development, based on the observable activity of children. Actions are assumed to induce the creation of schemes, such as producing counted items by pointing the finger to the table as many times as there are items not visible (in order to determine the quantity). The child 
is further supposed to create a feedback system (keeping track by creating finger patterns) when the task is not solvable with existing strategies or schemes. Development occurs from within the child, and it is assumed that a teacher can only indirectly bring development forward through presenting problems for the children to solve (Steffe, 2004).

A typical progression in children's counting skills starts, according to Clements and Sarama (2011), with acquiring the concept of cardinality, which they describe as expressed when children connect their counting on the number sequence with a subitised ${ }^{5}$ set of items. Number words in the counting sequence are ordinal in meaning, and subitizing is by definition cardinal. This way of describing a path in developing counting skills emphasises that children should learn to count on the number sequence and then relate the counting action to an experienced (cardinal) set that confirms the meaning of the last uttered number word. In Gelman and Gallistel's seminal work (1978), this is known as acquiring the cardinality principle, which is one of five counting principles that guide the acquisition of the verbal counting sequence. However, Gelman and Gallistel take a different standpoint in how the acquisition happens. They claim, "A knowledge of counting principles forms the basis for the acquisition of counting skills" (p. 204), meaning that principles are there ready to be applied by the child, which they interpreted based on observations of children's own logic, such as making up a stable counting sequence but in an unorthodox order.

Central to the research referred to above is to determine what skills are significant for successful arithmetic problem solving and what strategies children can learn to master. However, interventions in early years show ambivalent results, as it seems difficult to teach young children strategies that will be used in proficient ways. Or in other words, to facilitate their constructing arithmetical meaning and strategies. For instance, it has been shown that preschool children can learn to identify the inverse feature of addition and subtraction when presented a task with concrete manipulatives, but they do not make use of the principle in formal arithmetic problem solving (Nuñes et al., 2012). Thus, learning to apply strategies in problem solving is a delicate task and seems to remain instrumental in many cases, as observations of difficulties in transferring number facts to larger number ranges and novel tasks has shown (Björklund \& Runesson Kempe, 2020). This is why we are attempting to see elementary arithmetic development in light of an alternative approach, to enlighten our understanding of what is to be learnt in order to develop an understanding of the arithmetic strategies, which are also applicable in novel situations.

\section{Aspects known to be key to arithmetic skills}

Research agrees that there is more to solving arithmetic tasks than learning procedures and that certain attributes of numbers are recurring as central (such as ordinality, cardinality, number relations and representations). Learning to use numbers fluently in arithmetic tasks is claimed to presuppose an awareness of numbers' dual meaning of ordinality (following in a consecutive order) and cardinality (referring to a composed quantity), which for instance Fuson (1992) has taken as a point of departure for explaining necessary abilities for advancing in the use of strategies (in terms of starting from a counting act or starting from a set with cardinal

\footnotetext{
${ }^{5}$ Subitizing means the cognitive ability to instantly recognize an exact number of items without counting, described by Kaufman, Lord, Reese and Volkmann as early as 1949, and in infants and young children by Wynn (1998).
} 
value). Cardinality has been studied across cultures and languages, ending up in a consolidation of it being one of the core components of numbers and an awareness that develops similarly with differences only found due to children's linguistic environments (e.g., Le Corre et al., 2016; Sarnecka et al., 2018; Sarnecka \& Lee, 2009). Young children have thus been observed learning the cardinal meaning of "one", followed by "two" and "three" in consecutive order, until the subitizing range is extended and they comprehend that any number word relates to an exact quantity. According to Variation theory, however, "one" is the "manyness" of single objects, a shared attribute of every single object there is. This means that a child cannot initially look at something and discern its attribute of being one, and nor can they see that two or more things have this attribute in common. On the other hand, they would probably be able to see the difference between two things and one thing of the same kind (i.e., subitizing). Thus, the child must encounter at least two different numbers in order to discern the meaning of numbers. The implications of the Variation theory of learning is thus in conflict with the way of describing the development of cardinality awareness above. At this point, we suggest the conflict as a topic for future research.

Recently, attention has also been directed to ordinality as another core aspect of numbers that needs attention (e.g., Baccaglini-Frank et al., 2020; Sinclair \& Coles, 2017). Brissiaud (1992) described a specific way of using number words that is found in observations of young children, so-called word tagging (linking number words to objects one-to-one) in which the ordinal aspect of numbers is prominent (see also Neuman, 1987). But Brownell (1935, p. 21) concluded much earlier: "There is no quantitative significance in such counting; the child might as well be saying, 'a, b, c, d, e,' as ' $1,2,3,4,5$.' 'This is close to the meaning of ordinal numbers, whereby "five" does not mean the group of counted items but rather the last (fifth) one. If "five" indeed refers to a group of items, it is constituted of discrete ones, i.e., one, and one more, and one more, and one more, and one more. Piaget (1952) argued that this is the first sense of an arithmetic principle (primitive addition) that children express as early as their second year of life. Since the counted "ones" form a very loose organization, this way of experiencing numbers will however not help the child determine the quantity of a set or, for that matter, how to find a sum or even a missing addend.

Studies of intervention programmes have shown that it is possible to teach young children to handle novel and more advanced arithmetic tasks based on knowledge of numbers' partwhole relation (Fischer, 1990). Some researchers advocate that a structural approach, which primarily directs attention towards relationships between elements (Venkat et al., 2019) and making use of part-whole relations rather than single unit counting strategies (such as "counting all" and "counting on"), should be emphasised already in the early years (Brissiaud, 1992; Brownell, 1935; Davydov, 1982; Neuman, 1987; Schmittau, 2004). One argument is that single unit counting strategies may solve simple arithmetic tasks but do not support children's potential to recognise the part-whole structure that more advanced tasks presuppose. Recognizing and making use of number structure is a complex task itself, however. It builds on the child experiencing numbers as composite sets, which does not appear by counting single units, for instance on the fingers. However, knowing what different numbers look like in terms of finger patterns may emphasise, for example, the idea of numbers being parts of larger numbers and that numbers can be partitioned into smaller numbers in different ways (see Brownell, 1935; Björklund et al., 2019). Numbers are then represented by fingers, again in different ways, and a task can be treated as a part-part-whole relation. Counting fingers as single units and using finger patterns to represent numbers are thus two entirely different things, with radically different effects on learning. Research does 
convincingly show that certain aspects are keys to develop arithmetic skills and some advocate approaches that emphasise number relations as a way to support children in learning sustainable and developable strategies (Ellemor-Collins \& Wright, 2009; Resnick, 1983). Nevertheless, how these aspects are to be interpreted as component parts of arithmetic skills development is still in its infancy.

\section{The study}

In this paper, we present an inquiry into aspects that are necessary to discern for developing elementary arithmetic skills (what is to be learnt), and what it takes to develop these skills in early years (how this might be learnt) taking our point of departure in an approach to research that is theoretically grounded in the Variation theory of learning, discussed briefly in the introduction. The inquiry is based on empirical data from interviews with 4- to 7-year-olds.

\subsection{Empirical data}

Our study is meant to contribute to knowledge of arithmetic development, with conclusions drawn from empirical analyses of preschool children's number knowledge. The analysis is carried out on data from the research project FASETT. In our research project, we have conducted task-based interviews on three occasions (interview 2 with an eight month delay and interview 31 year after the second interview) with 103 children in the age span 58 months (4 years 10 months) to 89 months (7 years 5 months).

The children's legal representatives have given their informed consent for the children's participation and video documentation of the interviews. When consent for video documentation was not given, the interviews were only audio-recorded and field notes were taken to ensure proper documentation of the children's ways of handling the tasks both verbally and bodily (e.g., using their fingers). The interviews were task-based, including eight addition and subtraction tasks within the number range 1-10 (see Appendix). All tasks were given verbally, and no manipulatives were available except for the child's own fingers ${ }^{6}$. The interviews were conducted individually at the children's preschools by the project researchers. The tasks were the same on all three occasions, but due to the long intervals between the interviews it was found unlikely that children would have learnt the tasks from a previous interview occasion. The interviews focused on children's ways of handling the tasks, that is, how the children came to an answer. For this purpose, they were encouraged to explain in words and actions what they did and how they reasoned in their attempts to solve the tasks.

The data for analysis consist of 2184 observations of the children solving arithmetic tasks (observations in which children made no visible attempts to solve a task were excluded). An observation constitutes the child being given a task to solve and the child's response to this particular task. The children's responses in these interviews have been analysed with different focuses, in terms of qualitative changes over time (Björklund et al., 2021) as well as quantitatively (Kullberg et al., 2020) to describe learning outcomes. The analysis in this

\footnotetext{
${ }^{6}$ No numerals in writing were used during the interviews. In the description that follows in the article, numerals are used in the text to help the reader keep track of which task it is.
} 
particular paper adds to the previous studies by focusing on the aspects discerned and what they mean for arithmetic development.

\subsection{Methods for identifying critical aspects}

The question we raise is what is to be learnt, or more specifically, what aspects have to be discerned in order to develop arithmetic skills? To find the critical aspects in children's arithmetic problem solving, the units of analysis were the instances of children's ways of handling numbers, shown in both verbal utterances and gestures (such as finger patterns). The fundamental principle in our way of analysing is based on the Variation theory of learning (Marton, 2015), in that the combination of the necessary aspects for handling numbers in an arithmetic task discerned by a particular child defines their way of experiencing numbers. To solve an arithmetic task, some aspects are prominent and necessary to experience while other tasks demand other (or more advanced) aspects to be experienced. When a new dimension of variation is opened up, it "liberates" a new way of experiencing numbers and thus what the child can do with numbers. In our analysis, we thus look for what aspects the child expresses in their ways of handling a task, and we direct particular attention to which aspects the child who fails to handle a task has not yet discerned.

It is more difficult to find the necessary aspects if none of the observed children show it, or in fact also if all of them show it. For instance, cardinality is one such aspect that most 5-yearolds show to be experienced in when handling numbers. However, when a child does not show an awareness of the cardinal meaning of numbers this stands out as critical (against the background of all the "cardinal knowers") and is recognised, as it has an impact on what the child is able to do with numbers: expressing a different way of experiencing numbers in that the numbers, to the child, lack the meaning of "manyness". This means that the likelihood of discovering a certain aspect varies with the different groups of children that are observed. It also means that we can never be sure of having discovered all the necessary aspects and that there always remains the possibility of finding new ones. Nonetheless, we mostly find the same necessary aspects when children of similar age, living under similar social and cultural conditions, face the same tasks, in this case Swedish preschoolers from mixed socio-economic backgrounds ${ }^{7}$.

\subsection{Analysis}

We first conducted qualitative analyses of each observation to determine how the numbers in the presented tasks were experienced, in terms of which aspects the child discerned and which ones did not come through in the observation. We then differentiated observations of successful arithmetic problem solving (producing correct answers) and the less successful (producing incorrect answers). In the large sample of observations, we thus had a spectrum of correct and incorrect answers relating to discerned aspects, covering all the tasks in the interviews (presented in Table 1). We thereby found

\footnotetext{
${ }^{7}$ In Sweden, preschool is a non-mandatory pedagogical practice for children aged one to five years, with no standards for knowledge to achieve but only mathematical areas to offer children the possibility to explore and develop their knowledge within play-based settings. The year children turn six, they attend (at the time of this study, still on a voluntary basis) preschool class, a preparatory year before compulsory education commences.
} 
empirical indications of what aspects were necessary to discern in order to solve certain tasks. These aspects, and their relation to success or failure in arithmetic problem solving, are the main outset for further inquiry into what the discernment of certain aspects means, in line with the theoretical conjectures we outlined earlier.

\section{Relationships between critical aspects of numbers found in the present study}

Four main categories of necessary aspects of numbers were found in the analysis of the empirical data: modes of number representations, ordinality, cardinality, and part-whole relation. The part-whole relation aspect is further divided into four subcategories: differentiating parts and whole, decomposing numbers, commutativity, and inverse relation between addition and subtraction. In the arithmetic tasks, these are important to attend to, but above all they are discerned by the children when they are opened up as dimensions of variation (see Björklund et al., (2021) for qualitative examples of learning outcomes, and Kullberg et al., (2020) for quantitative proof of learning outcomes).

The aspects observed in our study are known in the field of research on learning elementary arithmetic. However, in our study we are able to conclude how these aspects relate to one another as components of the child's way of experiencing numbers, which limits or opens up the possibilities for them to apply different (or rather more or less powerful) strategies in their arithmetic problem solving. Categories A-D below refer to the aspects found in our study and the ways in which they are discerned by the children. Table 1 presents the frequency of correct and incorrect answers in relation to which aspects are discerned (and not discerned).

A. The child experiences only the modes of number representations (numbers are related to verbal expressions or representations with symbolic items such as fingers).

B. The child experiences the ordinality of numbers (numbers built as a sequence of single units), including modes of representations but not cardinality.

C. The child experiences the cardinality of numbers (numbers constitute composite sets), which includes modes of representations but not ordinality.

D. The child experiences numbers' part-whole relation (structuring tasks and relating numbers to other numbers in the task), which includes experiencing modes of number representations, ordinality and cardinality. This further includes:

\section{D1. Differentiating parts and whole \\ D2. Decomposing numbers \\ D3. Commutativity \\ D4. Inverse relation between addition and subtraction}

Our analysis of discerned aspects and observations of correct/incorrect answers confirms observations and conclusions in earlier research that being able to attend to the part-whole relation of numbers (category D) entails more successful arithmetic problem solving (see Table 1). It also confirms earlier claims regarding the necessity of coordinating cardinal and ordinal aspects of numbers, as our observations show that children only discerning the ordinality (category B) or the cardinality of numbers (category C) rarely solve the arithmetic tasks. 
Table 1 Categories of discerned aspects and the relation to correct and incorrect answers

\begin{tabular}{llll}
\hline & Correct & Incorrect & Total \\
\hline A. Modes of number representations & 1 & 25 & 26 \\
B. Ordinality & 15 & 123 & 138 \\
C. Cardinality & 37 & 580 & 617 \\
D. Part-whole relation & 1145 & 155 & 1300 \\
No answer & - & - & 103 \\
Total & 1198 & 986 & 2184 \\
\hline
\end{tabular}

What is striking in our observations is that even when children are observed to handle numbers' part-whole relation (category D), many incorrect answers are nevertheless given (12\% incorrect). This opens the opportunity for further inquiry into how the discernment of the aspects opens the way for more powerful strategies to be enacted, and particularly for taking a closer look at the construct of experiencing numbers' part-whole relation, as it seems to be a complex aspect. Thus, the aspect of part-whole relation includes subcategories that emerge as critical in some tasks.

How the aspects and subcategories are discerned (or not discerned) as dimensions of variation, presented in the following sections, will show why a certain aspect is necessary to experience, concluding in a discussion of how these aspects might be learnt.

\subsection{Modes of number representations}

Numbers are typically represented by young children using fingers or number words. However, if verbal representations are the only mode that numbers are represented in, the numbers are seen as only a certain kind of words. For example, one child was asked to figure out how many marbles (out of seven) the experimenter had hidden in her two hands (Task a). The child suggested "twenty here" and "twenty-one there" without further explanation. This observation implies and confirms earlier findings (see Fuson, 1992) that children usually learn that there are different number words before learning how to use them in a quantitative sense. But they certainly use them in some sense, and can distinguish number words from non-number words within certain limits. Another mode of representing numbers common in the early years is fingers. However, knowing that "five" can be shown by a whole hand does not necessarily mean it represents a quantity; it can be learnt as illustrating an image of a (number) word (Björklund \& Reis, 2020). Thus, discerning modes of representations is necessary, particularly for experiencing how different modes of representations apply to the same number - saying "five" and showing five fingers and pointing to a set of five marbles (see Björklund et al., 2021). However, discerning different modes of representations is not sufficient for solving arithmetic tasks, or for determining any set of objects as a quantity. On the other hand, it shows an important principle of Variation theory: opening up a critical aspect of the object of learning amounts to the simultaneous awareness of different features (e.g., different modes of representations) related to the same referent (in our case, number). Otherwise, if two features are only experienced together, they cannot be separated from each other: in that case, the child would have no concept of number nor of modes of representations.

\subsection{Ordinality}

Most of the 4- and 5-year-olds in our study could recite the counting rhyme up to 20 or higher. Thus, they at least experience numbers as ordered series of words. Ordinality implies that every 
number word has its exclusive place in the sequence (Gelman \& Gallistel, 1978). Some children make use of their fingers to secure the sequence, meaning that they always start counting from one finger and continue along the fingers and on the other hand in a stable order. This would be an example of a child at the same time discerning modes of representations (fingers and counting words) and ordinality. Still, these discerned aspects are not enough to allow the child to determine quantities of sets. When asked to show "six fingers", the child might show their right thumb alone (the sixth finger). This is an example of the aspect of ordinality being restricted to the place in a predetermined order, meaning that "six" indeed follows "five" and precedes "seven" but does not have a cardinal meaning.

The following excerpt is one example of how the ordinal aspect of numbers only, but not the cardinal aspect, is shown in a child's arithmetic act when encountering a task of seven marbles hidden in the interviewer's two hands and are asked to determine how the marbles can be divided between the two hands (Task a):

\section{Excerpt 1}

Mary: Seven in this (pointing at the interviewer's closed right hand)

Interviewer: And how many in this, then (left hand)?

Mary: Six

Interviewer: Okay, then, what were you thinking when you said it was seven in this and six in that?

Mary: Why... it's like ... when you count, it's first six and then seven.

We suggest that Mary saying "when you count, it's first six and then seven" is an indication that she perceives the ordinal aspect of numbers, but not the "manyness" of seven. The marbles called "the one, the two, the three... the six" are thus in one hand, and the marble called "the seven" (or the 7th marble) is alone in the other hand. The different aspects discerned as dimensions of variation constitute the ordinal meaning of consecutive numbers. These features are the functions of the preceding and following numbers. Discerning the ordinal aspect of numbers without simultaneously discerning their cardinal value, however, implies a serious limitation when it comes to handling quantities.

\subsection{Cardinality}

Fundamental for arithmetic problem solving is the idea that, for example, three items of any kind can be counted but have the same numerical meaning as any other set of three items: the cardinality of numbers. Three raised fingers may then represent the number, and can be manipulated as a number in the same way a set of marbles can be manipulated (here the modes of representations are present and help the child in discerning cardinality as a dimension of variation). The manipulation is not directed towards the items themselves, but what they represent.

An awareness of cardinality is observed in our study, with some children giving a plausible answer when encountering simple addition tasks but not seeming to relate numbers to other numbers (which would indicate a sense of ordinality). For example, Excerpt 2 shows a child suggesting two sets that are close to a plausible answer (the same Task a as in Excerpt 1 above), but without justifying any relation between the sets and the whole, still expressing some sense of the cardinality or "manyness" of numbers: 


\section{Excerpt 2}

Kim: [points at the closed right hand] One in that one [points at the closed left hand] five in that one.

Interviewer: How did you figure that out?

Kim: [points at the left hand] Eight in that one and two in that one [points at the right hand].

The child in Excerpt 2 does seem to discern that numbers have a meaning of "manyness" (cardinality), but since no other aspect that would direct attention to the relations between numbers in an ordinal sense are discerned, the child only solves the arithmetic task as approximate.

When children discern the cardinal aspect of numbers without discerning their ordinal aspect, they might be able to grasp the "manyness" only of small and approximate quantities but still cannot enact any counting strategies that could ensure them of how a set of items constitute units that can be added, subtracted, divided, or compared. What we see in our observations is that when children simultaneously discern the ordinal and cardinal aspects of numbers they often manage to solve straightforward addition and subtraction tasks $(2+5=$ Task e, and $10-6={ }_{-}$Task f). However, we suggest that missing addend tasks and whole unknown tasks $\left(3+_{-}=8\right.$ Task $g$, and ${ }_{-}-3=6$ Task $\left.\mathrm{h}\right)$ require still other aspects to be discerned. Thus, it is not enough to coordinate the ordinal and cardinal meaning of numbers. We found a critical aspect to be the part-whole relation as well, which enabled the children to solve the more complex tasks.

\subsection{Part-whole relation}

In our study, we find that missing addend and start-unknown tasks $\left(3+_{-}=8\right.$ Task $\mathrm{g}$, and $\_-3$ $=6$ Task $\mathrm{h}$ ) require the child to see numbers as related to the other numbers in the task, in order to enact a suitable strategy. That is, the part-whole relation aspect becomes critical to many of the children. When they do not discern this aspect, they are forced to keep track of counted single units. For example, solving the task "You have three glasses, but are going to set the table for eight people, how many more glasses do you need?" (Task g) is easy if the child discerns the parts as three, one unknown and the whole eight, thus seeing the three in the eight and the missing number as the other part. However, if the part-whole relation of the numbers is not discerned, children are observed keeping track of how many single units have been added, for example: " 3 , that's 4 ( 1 added), 5 ( 2 added), 6 ( 3 added), 7 (4 added), 8 (5 added)". This "double counting" (see also Neuman, 1987) reflects the child alternating between foregrounding the ordinality (a consecutive order of counting words) and the cardinality (how many glasses there are for each added one). Thus, the child has to work with parallel number sequences, which often results in incorrect answers or the child losing track of the units counted (see Björklund, 2021 for a deeper inquiry).

The finding that strategies building on a part-whole relation between numbers are successful is not new; the same conclusion has been drawn in other empirical research as well (e.g., Baroody \& Purpura, 2017; Ellemor-Collins \& Wright, 2009; Schollar, 2015). Nevertheless, surprisingly many children who seem to discern number relations while trying to solve the tasks end up with incorrect answers (see Table 1, category D). Our qualitative analysis of what aspects children discern when solving arithmetic tasks can however shed light on this outcome: 
discerning the part-whole relation of numbers is important, but not sufficient, it constitutes in itself several aspects that are necessary to discern. We will elaborate on how we came to this conclusion in the discussion below.

What the part-whole relation constitutes is generally that numbers are perceived as composite sets that relate to other sets, which makes it possible to treat numbers in arithmetic tasks as relational. Depending on the encountered task, certain features of the part-whole relation become necessary to discern, such as differentiating parts and whole, decomposing numbers, commutativity, and the inverse relation between addition and subtraction. Not all of these aspects are necessary to discern in order to solve all kinds of arithmetic tasks in this study; some of them become critical in solving certain tasks, but are not necessary for solving others.

\subsubsection{Differentiating parts and whole}

Many arithmetic tasks are quite straightforward, particularly those that preschool children encounter in daily interaction (adding two sets or removing a part from a larger set). However, in our interviews we found it to be critical for children to specifically differentiate parts and whole in such tasks that asked for a missing part. The differentiation of parts and whole is closely connected to the task at hand. For many children, it was difficult to discern the partwhole relation in the task "You and your friend collected five shells together, you collected four of them, how many did your friend collect?" (Task c). Many children came up with the answer nine; that is, adding five and four. It was observed that children who did distinguish parts and whole related the given four to the larger number five, and either counted on "one" or structured the task on their fingers, starting with the whole five by unfolding five fingers and then folding four to see the remaining unfolded one (usually the thumb) constituting the missing part. Thus, the part-whole relation includes this aspect as necessary in order to understand the number structure in this and other arithmetic tasks.

\subsubsection{Decomposing numbers}

Children who discern the decomposition of numbers are often capable of solving the tasks in our study, and often use finger patterns to structure the numbers. However, recognizing a pattern in quantitative terms entails perceiving how numbers constitute composite sets of units and how they can be composed and decomposed in different ways to ease the computation process. Some ways of composing numbers are more powerful than others: "You have three glasses on the table but are going to set the table for eight people. How many more glasses do you need?" This task (Task g) can be solved in several distinct ways, either by creating the given part three on one hand (three unfolded fingers) and unfolding the five fingers on the other hand to make the whole finger pattern of "eight" and recognizing the whole hand "five" as the missing part, or by creating the given part "three" on one hand and adding on the following two fingers and three more on the other hand while counting "three... four, five, and six, seven, eight" and then seeing the two and three as the missing five, or by starting with composing the whole as two sets of four unfolded fingers, then folding one finger and saying "three" while unfolding another finger on the other hand, thus showing a whole hand as "five". All of these ways of composing and decomposing numbers are observed in our study (see also Kullberg \& Björklund (2020) for a discussion of the developmental potential of the different finger patterns used). 
The aspect of decomposing numbers is discerned by children who know numbers as number facts and thus express a fluent use of numbers. Their way of experiencing numbers' part-whole relation makes them able to operate on, or simply "know" the answer to an arithmetic task, which allows them to use more economical strategies as this induces derived facts and numerical transformation (Gray et al., 2000). One illustration of this from our study is a child solving the task "If you have ten candies and eat six of them, how many are left?" (Task f), who gave an answer of 4, describing how he decomposed 10 into 5 and 5 and then moved 1, saying, "Move over one from five, then there are four left." In this case, the dimension of variation is comprised of different ways of dividing the whole (10). One way is " $10-6=$ _" while another, expressed by this child, is " $10=5+5$ and $5+1+4=6+4$ ".

\subsubsection{Commutativity}

Included in the aspect of the part-whole relation of numbers we find the principle of commutativity. Indeed, commutativity makes an arithmetic task easier when the child is aware of the efficacy of adding the smaller part to the larger one, but when this is seen only as a procedure there is a risk of misusing the strategy (such as applying it to subtraction). We prefer to say that children have opened the ordering of addends dimension of variation when they discover that the order of the addends does not have to be taken for granted, they can be switched in an addition task, so you can always start with the largest part. If the child does not experience numbers' part-whole relation, switching addends is not an option. The part-whole relation also constitutes the aspect of commutativity as one to be discerned in certain tasks (but not necessarily all).

By opening up commutativity as a dimension of variation the child sees, for instance, Task e as " $2+5$ or $5+2$ " instead of a static expression " $2+5=$-" whereby one has to start with the first presented addend. Discerning commutativity induces a way of experiencing numbers' relations, for example that the parts are perceived as composite sets that can be shown as finger patterns ( 2 on one hand and 5 on the other, together recognised as the pattern for 7). Some of the children in our study were observed spontaneously opening this dimension of variation by crossing their hands while retaining the finger patterns 2 and 5 .

We suggest that commutativity is an essential feature of the part-whole relation of numbers, as it presumes the child experiencing the parts as simultaneously constituting the whole; then, it does not matter to the child whether 5 is added to 2 , or 2 is added to 5. In our study, some children explain their making use of the discerned commutativity by saying "it's easier that way", as the child then only has to add on two units, which are easily subitised either by hearing the number of counted number words ("five....and six, seven") or by raising one's fingers to keep track. To conduct this operation, the child focuses on both the cardinal and ordinal aspects simultaneously and, in addition, perceives the number relations as equivalent. If the ordering of addends is taken for granted, or in our terms is undiscerned, the child counts on "two... and three, four, five, six, seven...", which was observed to result in the child not knowing when to stop adding units (because the subitizing range is transcended). The only solution children then find is to keep track of the added units by means of their fingers, which itself becomes a problematic strategy as the number range increases. Thus, discerning commutativity supports the awareness of the number relations given in a task. 


\subsubsection{Inverse relation between addition and subtraction}

In our study, we observed that the inverse relation between addition and subtraction as a feature of the part-whole relation becomes critical when children solve the verbal task "You have ten candies and eat six of them, how many are left?" (Task f). This task can be solved as either $10-6=\ldots$ or $6+_{-}=10$. Some children solve the task by counting down, which calls for the rather complicated double counting (keeping track) strategy. Others change the operation of the task by handling it as an addition task by saying " $6 \ldots 7,8,9,10$, it's 4 ", hearing through what we can call "auditory subitizing" that four units have been added, or by keeping track with fingers. Having discerned the inverse relation as a dimension of variation (choosing between subtracting or adding, or between "going down" or "going up") concerns the alternatives "seeing subtraction as subtraction" or as "addition or subtraction", the latter enables the child to solve subtraction tasks in more flexible ways.

In fact, this feature of the part-whole relation does not become critical at all to some children, who rather perceive an arithmetic task as a part-whole relation in which some addends (at least one) are known; sometimes the whole is known, and sometimes the whole is what is asked for. The children then do not distinguish between addition and subtraction, but rather see them as two sides of the same coin.

\section{Revisiting the conjectures}

As we mentioned above, what we aim to contribute through this paper is a way to describe and understand arithmetic development in the early years in terms of discerned critical aspects, with implications for what should be taught to children to facilitate their development of arithmetic skills. We have presented four primary aspects found empirically in a study with four- to seven-year-olds, the last of which includes four subcategories. In the following, this description is seen through the lens of the five conjectures we outlined above as some of the main principles in our theoretical framework. When presenting these conjectures we explained that our intention with the present work was to further support those conjectures. What has been added is - as depicted in the set of five conjectures - that we can identify for any group of young children a set of critical aspects that they have to master in order to make the object of learning (in our case the numbers 1-10) their own. What is meant to be our major contribution to the field is the identification of the critical aspects of the first ten numbers in the context of elementary arithmetic and how they relate to one another as attributes of arithmetic skills. Let us now revisit the five conjectures.

First, differences in young children's ways of handling arithmetic tasks derive from differences in their ways of perceiving these tasks.

The first conjecture suggests that a difference between two ways of handling a task implies a difference between two ways of perceiving it. Our study of children's ways of handling the same tasks provides multiple examples of the differences between children's ways of perceiving numbers. For example, in a task in which the start number is unknown ( $-3=6$ Task h), we see some children claiming that the task in which some items are removed (broken) and some remain is not possible to solve, as they do not know the initial number of balloons that is asked for and thus cannot make sense of the task. Other children instantly recognise the task as 
an addition problem, seeing the broken items and the remaining ones as parts of one whole, and can easily solve it. Another example that illustrates this conjecture is when the children encounter subtraction tasks, such as "You have ten candies and eat six of them, how many are left?" (Task f): some children see the task as "subtraction" and count down from ten, while others see it as "subtraction or addition" and in this case often choose to count up from the given part. The latter way of seeing the task enables the child to solve subtraction tasks in ways that are more flexible.

Thus, we claim that this distinction is based on the different ways of perceiving numbers, which makes some strategies for solving a task possible to enact and others impossible because they do not make sense to the child. The interpretation that differences in children's ways of handling arithmetic tasks reflect differences in their ways of perceiving those tasks appears thus reasonable.

Second, differences in young children's ways of perceiving arithmetic tasks derive from which aspects of these tasks they simultaneously discern and focus on.

In order to experience numbers in ways that allow the children to solve arithmetic tasks, they have to discern and focus on several aspects simultaneously. The four aspects found in our empirical investigations have shown to be critical for children's abilities to solve arithmetic tasks. However, if only the first three (the cardinal, ordinal, and representational aspects of numbers) are discerned irrespective of each other, the child is unlikely to solve the arithmetic task. Only when the three aspects and the part-whole relation aspect are discerned simultaneously does it become possible for the child to enact strategies that help in solving the arithmetic task.

For instance, children often solve the task "You have two sea shells and your friend five; how many do you have together?" (Task e) by showing the finger pattern five on one hand with all fingers unfolded and two fingers on the other hand. Some children in our study add "Seven, we have seven together", which indicates that they see numbers as composite sets and have discerned a part-whole relation. Other children add to their finger pattern the verbal answer "Two and five; I have two and she has five" and fail to see them as constituting a composite whole. Our interpretation is then that the latter utterance is an expression of the child not discerning a part-whole relation of numbers, as the parts five and two are not seen as being included in a larger set. Therefore, even if children seem to express the same number pattern as an answer to a task, the meaning behind the finger patterns can be very distinct. This, we suggest, is because they experience different aspects of the task, which defines what they find possible to do with the numbers in it. Furthermore, depending on the task at hand, certain features of the part-whole relation may also be foregrounded that allow the child to enact more powerful strategies, such as seeing the commutativity aspect of the task and thus adding two to five even if the task was presented as having two and then five more. Which aspects of a task that are discerned and focused on simultaneously reflect differences in young children's ways of perceiving (and thus handling) the task.

Third, an aspect of an arithmetic task is a dimension in which commensurable (comparable) attributes may vary in the learners' awareness.

Each aspect constitutes a dimension that is essential to experience for the aspect to become present for the child. This can be seen, for instance, in the fact that the aspect modes of number 
representations is vital in many arithmetic problem solving situations. The child is supported in encountering the task by discerning how different modes of representations, such as verbal counting words and finger patterns, represent the same number, thus offering the child an opportunity to structure number relations on their fingers, to ease the problem solving. Another example is the discernment of the cardinality or "manyness" of numbers, which means seeing numbers as composite sets, which can vary between sets, thus relating numbers' cardinal meaning to other numbers' cardinal meaning as dimensions of variation. It is thereby necessary for the child to see, for instance, how cardinality is a common feature of all numbers or, for instance, the part-whole relation as a feature that is applicable to all arithmetic tasks.

Fourth, aspects are "critical"; i.e., necessary to discern in order to enable the learner to solve certain kinds of arithmetic tasks.

Children may fail to solve a task because they fail to discern one or several aspects of it. This conjecture becomes essential in our study of what is to be learnt, as it tells us what children are and are not able to do with an arithmetic task. Aspects are critical to the child, because discerning this critical aspect enables them to see the arithmetic task differently and thus enact different strategies to solve the problem. If the part-whole relation aspect is not experienced in terms of differentiation of parts and whole, some tasks become error-prone, such as adding given numbers in a task even though it is a missing addend that is asked for $(5=4+$ _ Task c $)$. Similarly, many subtraction tasks are easily solved when the child discerns the inverse relation between addition and subtraction. When this aspect is not discerned, many are forced to apply double counting strategies, which often result in incorrect answers. For these children, the inverse relation between addition and subtraction is critical to discern in order for powerful strategies to be applied in the problem solving.

Fifth, teachers can help learners to open up critical aspects by bringing about patterns of variation and invariance.

Situating our examples in the discourse of research on learning and teaching early mathematics, our findings offer a way of describing the teaching-learning relation in commensurable terms, that is, aspects found critical for learning arithmetic skills are exactly what teaching should enable the child to discern as dimensions of variation (Björklund et al., 2021). Thus, a unique opportunity is afforded to differentiate what should be learnt as well as how this learning could be enabled by our framework.

Teachers can help learners to open up critical dimensions of variation by bringing about patterns of variation and invariance. The examples and conclusions shown above illustrate how differences in perceiving numbers (and thus what can represent numbers and what to do with numbers) are functions of differences in which aspects are discerned by the child in a particular situation (Conjecture 1). Some tasks bring to the fore the necessity to discern several aspects and the child's ability to experience these, simultaneously, also constitutes their way of perceiving and solving the task (Conjectures 2, 3 and 4). As our study reveals, which aspects of numbers are discerned in a particular problem solving situation has a significant impact on the strategies children enact and thus on their success in solving the task. What teaching should attend to, then, is to allow necessary aspects to be discerned by the child, by offering patterns of variation that allow the child to see the arithmetic task in a new and different way (Conjectures 3 and 5). Interventions conducted according to these principles of teaching, in 
this case emphasizing the part-whole relation aspect and its features, have proven to be successful, both in quantitative terms, producing more correct answers to elementary tasks (Kullberg et al., 2020) and in qualitative terms, developing the way of perceiving arithmetic tasks as constituting number structures to operate on rather than applying single unit counting strategies (Björklund et al., 2021).

As the prosperity of the implementation of the theoretical principles has been reported elsewhere, our attention here is rather on how the aspects-both empirically and theoretically — can be seen as related to one another in the development of arithmetic skills.

\section{What makes the learning of arithmetic skills possible}

In our efforts to answer the research question what is to be learnt, we have identified aspects necessary to discern in order to solve elementary arithmetic tasks. The validity of our inquiry, however, is rather dependent on whether we can argue for how the learning of arithmetic skills takes place - how it might be learnt - in terms of the discernment of these necessary aspects. In Table 1, we showed the frequency of correct and incorrect answers to the tasks in relation to what aspects a child is discerning (based on qualitative analyses of strategies and utterances of children's problem solving). Based on these empirical findings and the conjectures, we propose a description of children's advancement of arithmetic skills in regards to what dimensions of variation are necessary to be opened up.

In order to open up dimensions of variation (corresponding to critical aspect) the child needs to encounter at least two values in a particular dimension of variation. One of the aspects we presented as necessary for developing arithmetic skills - modes of representations — should then be opened up as a dimension of variation, by affording two different modes of representations for the same number, e.g., verbal expression and finger pattern, at the same time. If the child only encounters one kind of representation of one number (for instance, three fingers for "3"), they will not distinguish between the number and its representation. According to the Variation theory of learning, representations would not exist for the child in this case, it would not be possible to discern. When discerning this aspect, other necessary aspects are liberated as well. We argue that modes of representations become significant for discovering other necessary aspects of numbers. For instance, through the use of finger patterns, dimensions of variation may be discerned that bring to the fore the aspect cardinality (perceiving numbers as composed sets) and part-whole relation (see also Björklund et al., 2021).

Our description of arithmetic learning is however not strictly cumulative in nature. Our analysis shows that the children who only discern the ordinal or cardinal meaning of numbers have no ways of enacting arithmetic strategies, as they are not experiencing these aspects simultaneously. It is only when this dual meaning of numbers is attended to that they can enact basic arithmetic strategies. For example, children who are able to coordinate the cardinal and ordinal aspects of numbers usually end up with the correct answer in straightforward addition tasks $(2+5=\ldots$ Task e). However, some of them fail in solving tasks that require them to discern the part-whole relation, for instance responding to Task $\mathrm{g}\left(3+_{-}=8\right)$ : "Three, and four, five, six, seven, eight. It's eight!". This is a telling example of the children discerning the ordinal and the cardinal aspects, but not simultaneously, as the counting sequence only has one meaning at a time. Handling the numbers as either ordinal or cardinal will not illuminate number relations; the part-whole relation aspect is not present. In this particular example, the part-whole relation is a critical aspect to discern for finding the missing addend - seeing the 
three and the five in the eight. Thus, discerning more aspects liberates the child in seeing the task at hand in a more powerful way, which enables them to make a choice regarding how to encounter it.

A central point in our argument is thereby the need for the simultaneous discernment of several necessary aspects in order to succeed in arithmetic problem solving. In our study, the part-whole relation was found to be a complex of features that, when discerned, helps the child to see arithmetic tasks in ways that make them easier to handle. In some cases, one or several of these were even necessary in order to make sense of the task. This is particularly true when it comes to differentiating parts and whole, as this was found to be critical for many children in order to solve missing addends and start-unknown tasks (a, b, c, d, g and h).

Our way of describing the advancement of arithmetic skills in terms of discerned aspects makes it possible to explain why children cannot use certain strategies and how they may learn to solve tasks they could not previously solve. This is an alternative way of explaining how children learn (and what they need to learn) that is applicable in teaching (what dimensions of variation need to be opened up by the teacher). It even suggests that teaching strategies and procedures are insufficient if a child does not discern necessary aspects of numbers that would allow them to enact a certain strategy. Our conclusion is thereby that what children need to learn is not strategies for solving arithmetic tasks, but strategies that enable the child to see the tasks in a powerful way. There is thus a difference in learning to do and learning to see in order to do. We venture to suggest that this is the case, with support from quantitative analyses showing that children who are taught to experience numbers primarily as composite sets and to handle arithmetic tasks in accordance with numbers' part-whole relations significantly increase their success in solving tasks of different kinds with long-lasting effects, compared to children who have not participated in such interventions (Kullberg et al., 2020).

\section{Towards more powerful ways of seeing numbers}

We began this article by claiming that students differ in what they need to learn in order to achieve the objectives of a certain curricular unit. Throughout the paper, we have shown empirical examples of what children need to learn in order to develop their arithmetic skills, in terms of discerned aspects of numbers. Our line of reasoning, based on the Variation theory of learning, thereby proposes a complement to the existing theories in this field of knowledge.

How children learn to use strategies that are more advanced is a crucial question in our inquiry and there are many studies of children's strategy choice and execution of strategies in arithmetic problem solving (e.g., Carpenter \& Moser, 1982; Caviola et al., 2018; Cheng, 2012; Fuson, 1992; Gray, 1991; Nuñes et al., 2012). Ellemor-Collins and Wright (2009, p. 52) argue that adding and subtracting without counting is a critical goal in achieving children's numeracy, but that "Some students do not achieve this facility. Instead, they persist with strategies involving counting by ones for addition and subtraction in the range 1 to 20 , and in turn use counting strategies in the higher decades". How these students may learn to use more powerful strategies in arithmetic problem solving is thereby crucial knowledge for the development of mathematics education. Observations show, for instance, that counting by ones often entail double counting (when exceeding the subitizing range), a strategy in arithmetic problem solving which by Steffe (2004) is interpreted as the child having constructed a scheme of the number sequence that is supposed to bridge to strategic arithmetic reasoning. In our view, based on the Variation theory of learning, to encourage double counting as an arithmetic 
strategy cannot facilitate more advanced understanding since the strategy is founded on the perception that numbers are countable entities on parallel number sequences. What children need to learn is rather to perceive numbers in ways that emphasise the relational aspect of numbers (cf., Resnick, 1983). Double counting is in this sense not a bridge to more advanced understanding, it may instead lead to difficulties in arithmetic problem solving if the child is not afforded other, more powerful ways of perceiving numbers as relational (Neuman, 2013; Cheng, 2012).

Another main objective in early mathematics education is the development of the ability to retrieve known facts from memory; that is, instantly knowing the answer to an arithmetic task and handling numbers in a flexible way. In other words, children simultaneously recognise number relations and are able to decompose or regroup within and between numbers when applicable. Gray (1991) has pointed out that establishing knowledge of number facts goes beyond acquiring a mental repertoire of strategies. In fact, Gray suggests that remembering facts is less important than learning to design a method for how to solve a problem. In our terminology, this would entail the child learning strategies that would allow them to see the task at hand in more powerful ways. Nevertheless, also in line with Baroody (2016), for example, in solving arithmetic tasks that are more advanced it is important to have a conceptual understanding of number facts. This conclusion is based on our observations of children who see numbers as known facts opening up the possibility to decompose or make use of arithmetic principles such as inverse relations between addition and subtraction to transform the task into an easier one (see also Kilpatrick et al., 2001; Nuñes \& Bryant, 1996). This conclusion is also based on the theoretical frame that we propose, in that the skill to retrieve number facts from memory as a strategy to make use of known number relations constitutes a dimension in which comparable attributes vary in learners' awareness. However, recent analyses of children expressing having known number facts may in fact lack some discerned aspects that hinder them from practicing their skills when encountering for example, a larger number range (Björklund \& Runesson Kempe, 2020). This indicates that arithmetic skills are a complex of aspects to discern, some of which we have identified (for instance the features of the part-whole relation aspect, which is critical to discern depending on the task). More in-depth analyses of the construct of arithmetic skills are thus still needed.

In accordance with the above, powerful ways of handling elementary arithmetic tasks originate from powerful ways of seeing such tasks. Seeing a task in a certain way amounts to which attributes the learner simultaneously discerns and focuses on. The child's way of seeing the task, for instance the inverse relation between addition and subtraction, enables the child to solve subtraction tasks in flexible ways. This is observed many times in the large body of research on arithmetic skills, but our study may contribute a description of what the child has learnt when they are able to apply such strategies, and also how this knowledge might be learnt. The key is the directedness towards the child's way of perceiving numbers, and what aspects certain arithmetic tasks require the child to discern. The critical aspects we present in our study have certainly not been found here for the first time; all of them are known in the field of research on learning elementary arithmetic. However, they are interpreted and related to each other as "critical aspects" in the sense introduced in this paper. Critical aspects are specific not only to a certain object of learning but also to the specific group of learners participating in the actual study. This means that when it comes to a new object of learning or a new and specific group, like a preschool group, the most urgent and decisive pedagogical question must be to determine what the critical aspects of this specific object of learning are for 
this specific group of learners and how a child becomes aware of aspects that they have not discerned before.

\section{Appendix}

Tasks given in the assessment

a) 7=_+_ Seven stones are counted and then hidden in the interviewer's closed hands. How many can there be in the one hand and how many in the other hand. Multiple options possible.

b) $7=4+$ t The interviewer opens one of her hands, showing four marbles. If one knows there are seven altogether, how many are there in the closed hand?

c) 5=4+_You and your friend collected five shells together, you collected four of them, how many did your friend collect?

d) 9-7=_If you have nine shells and your friend has seven shells, how many more shells do you have?

e) $2+5=$ _ You have 2 sea shells and your friend 5, how many do you have together?

f) $10-6=$ If you have ten candies and eat six of them, how many are left?

g) $3+=8$ You have three glasses, but are going to set the table for eight people, how many more glasses do you need?

h) $\quad-3=6$ On the morning of your birthday party, you blew up balloons. At the party, three balloons broke, and there were only six balloons left. How many balloons did you blew up that morning?

Acknowledgements We wish to express our deep respect for and gratitude to Dr Dagmar Neuman. Our work is inspired by, and builds on, her inquisitive insights.

Funding Open access funding provided by University of Gothenburg. The study was funded by the Swedish Research Council (grant no. 721-2014-1791) during the period 2015-2018.

Open Access This article is licensed under a Creative Commons Attribution 4.0 International License, which permits use, sharing, adaptation, distribution and reproduction in any medium or format, as long as you give appropriate credit to the original author(s) and the source, provide a link to the Creative Commons licence, and indicate if changes were made. The images or other third party material in this article are included in the article's Creative Commons licence, unless indicated otherwise in a credit line to the material. If material is not included in the article's Creative Commons licence and your intended use is not permitted by statutory regulation or exceeds the permitted use, you will need to obtain permission directly from the copyright holder. To view a copy of this licence, visit http://creativecommons.org/licenses/by/4.0/.

\section{References}

Baccaglini-Frank, A., Carotenuto, G., \& Sinclair, N. (2020). Eliciting preschoolers' number abilities using open, multi-touch environments. ZDM-Mathematics Education, 52(4), 779-791. https://doi.org/10.1007/s11858020-01144-y

Baroody, A., \& Purpura, D. (2017). Early number and operations: Whole numbers. In J. Cai (Ed.), Compendium for research in mathematics education (pp. 308-354). National Council of Teachers of Mathematics.

Baroody, A. J. (2016). Curricular approaches to connecting subtraction to addition and fostering fluency with basic differences in grade 1. PNA, 10(3), 161-190. 
Björklund, C. (2021, in press). Children's awareness of numbers' part-whole relations when bridging through 10. In Proceedings from MADIF-12, The twelfth research seminar of the Swedish Society for Research in Mathematics Education, Växjö, January $15^{\text {th }}, 2020$.

Björklund, C., Ekdahl, A.-L., \& Runesson Kempe, U. (2021). Implementing a structural approach in preschool number activities. Principles of an intervention program reflected in learning. Mathematical Thinking and Learning, 23(1), 72-94. https://doi.org/10.1080/10986065.2020.1756027

Björklund, C., Kullberg, A., \& Runesson Kempe, U. (2019). Structuring versus counting - critical ways of using fingers in subtraction. ZDM-Mathematics Education, 51(1), 13-24. https://doi.org/10.1007/s11858-0180962-0

Björklund, C., \& Reis, M. (2020). Ways of using fingers in preschoolers' numerical reasoning. In M. Carlsen, I. Erfjord, \& P. S. Hundeland (Eds.), Mathematics Education in the Early Years. Results from the POEM4 Conference, 2018 (pp. 93-107). Springer.

Björklund, C., \& Runesson Kempe, U. (2020). Utveckling av räknefärdigheter hos 5-7-åringar - Matteuseffekt eller utfall av undervisning. [Development of arithmetic skills among 5-7-yearolds]. Forskning om Undervisning och Lärande, 8(1), 9-28.

Brissiaud, R. (1992). A tool for number construction: Finger symbol sets. In J. Bideaud, C. Meljac, \& J.-P. Fischer (Eds.), Pathways to number: Children's developing numerical abilities (pp. 41-65). Lawrence Erlbaum.

Brownell, W. (1935). Psychological considerations in the learning and the teaching of arithmetic. In Reeve (Ed.), The tenth yearbook. The teaching of arithmetic. The National Council of Teachers of Mathematics (pp. 131). New York: Teachers College, Columbia University.

Carpenter, T. P., \& Moser, J. M. (1982). The development of addition and subtraction problem-solving skills. In T. P. Carpenter, J. M. Moser, \& T. A. Romberg (Eds.), Addition and subtraction: A cognitive perspective (pp. 9-24). Lawrence Erlbaum.

Caviola, S., Mammarella, I., Pastore, M., \& LeFevre, J.-A. (2018). Children's strategy choices on complex subtraction problems: Individual differences and developmental changes. Frontiers in Psychology, 9(1209). https://doi.org/10.3389/fpsyg.2018.01209

Cheng, Z.-J. (2012). Teaching young children decomposition strategies to solve addition problems: An experimental study. The Journal of Mathematical Behavior, 31(1), 29-47.

Clements, D., \& Sarama, J. (2011). Early childhood mathematics intervention. Science, 333(6045), 968-970. https://doi.org/10.1126/science. 1204537

Davydov, V. V. (1982). The psychological characteristics of the formation of elementary mathematical operations in children. In T. P. Carpenter, J. M. Moser, \& T. A. Romberg (Eds.), Addition and subtraction: A cognitive perspective (pp. 224-238). Lawrence Erlbaum.

Ellemor-Collins, D., \& Wright, R. B. (2009). Structuring numbers 1 to 20: Developing facile addition and subtraction. Mathematics Education Research Journal, 21(2), 50-75. https://doi.org/10.1007/BF03217545

Fischer, F. E. (1990). A part-part-whole curriculum for teaching number in the kindergarten. Journal for Research in Mathematics Education, 21, 207-215.

Fuson, K. (1982). An analysis of the counting-on solution procedure in addition. In T. P. Carpenter, J. M. Moser, \& T. A. Romberg (Eds.), Addition and subtraction: A cognitive perspective (pp. 67-81). Lawrence Erlbaum.

Fuson, K. (1992). Research on whole number addition and subtraction. In D. Grouws (Ed.), Handbook of research on mathematics teaching and learning (pp. 243-275). Macmillan Library Reference.

Gelman, R., \& Gallistel, C. (1978). The child's understanding of number. Harvard University Press.

Gray, E., Pitta, D., \& Tall, D. (2000). Objects, actions, and images: A perspective on early number development. Journal of Mathematical Behavior, 18(4), 401-413.

Gray, E. M. (1991). An analysis of diverging approaches to simple arithmetic: Preference and its consequences. Educational Studies in Mathematics, 22(6), 551-574.

Kaufman, E. L., Lord, M. W., Reese, T. W., \& Volkmann, J. (1949). The discrimination of visual number. American Journal of Psychology, 62, 498-525.

Kilpatrick, J., Swafford, J., \& Findell, B. (Eds.). (2001). Adding it up. Helping children learn mathematics. National Academy Press.

Kullberg, A., \& Björklund, C. (2020). Preschoolers' different ways of structuring part-part-whole relations with finger patterns when solving an arithmetic task. ZDM-Mathematics Education, 52(4), 767-778. https://oi. org/10.1007/s11858-019-01119-8

Kullberg, A., Björklund, C., Brkovic, I., \& Runesson Kempe, U. (2020). Effects of learning addition and subtraction in preschool by making the first ten numbers and their relations visible with finger patterns. Educational Studies in Mathematics, 103(2), 157-172. https://doi.org/10.1007/s10649-019-09927-1

Le Corre, M., Li, P., Huang, B. H., Jia, G., \& Carey, S. (2016). Numerical morphology supports early number word learning: Evidence from a comparison of young Mandarin and English learners. Cognitive Psychology, 88, 162-186. https://doi.org/10.1016/j.cogpsych.2016.06.003 
Marton, F. (1981). Phenomenography - describing conceptions of the world around us. Instructional Science, 10(2), 177-200.

Marton, F. (2015). Necessary conditions of learning. Routledge.

Marton, F., \& Booth, S. (1997). Learning and awareness. Mahwah, N.J: Lawrence Erlbaum Associates.

Marton, F., \& Neuman, D. (1989). Constructivism and constitutionalism. Some implications for elementary mathematics education. Scandinavian Journal of Educational Research, 33(1), 35-46. https://doi.org/10. 1080/0031383890330103

Marton, F., \& Neuman, D. (1990). Constructivism, phenomenology, and the origin of arithmetic skills. In L. Steffe \& T. Wood (Eds.), Transforming children's mathematics education. Lawrence Erlbaum.

Marton, F., \& Tsui, A. (Eds.). (2004). Classroom discourse and the space of learning. Mahwah, NJ: Lawrence Erlbaum Associates.

Marton, F., Wen, Q., \& Wong, K. C. (2005). 'Read a hundred times and the meaning will appear ...' Changes in Chinese University students' views of the temporal structure of learning. Higher Education, 49(3), $291-318$. https://doi.org/10.1007/s10734-004-6667-z

Neuman, D. (1987). The origin of arithmetic skills. Acta Universitatis Gothoburgensis.

Neuman, D. (2013). Att ändra arbetssätt och kultur inom den inledande aritmetikundervisningen [Changing the ways of working and culture in early arithmetic teaching]. Nordic Studies in Mathematics Education, 18(2), 3-46.

Nuñes, T., Bryant, P., Evans, D., Bell, D., \& Barros, R. (2012). Teaching children how to include the inversion principle in their reasoning about quantitative relations. Educational Studies in Mathematics, 79(3), 371388. https://doi.org/10.1007/s10649-011-9314-5

Nuñes, T., \& Bryant, P. E. (1996). Children doing mathematics. Blackwell.

Piaget, J. (1952). The origins of intelligence in children. New York: International Universities Press.

Resnick, L. B. (1983). A developmental theory of number understanding. In H. Ginsburg (Ed.), The development of mathematical thinking (pp. 109-151). New York, NY: Academic Press.

Sarnecka, B., Negen, J., \& Goldman, M. (2018). Early Number Knowledge In Dual-Language Learners From Low-SES Households. In D. Berch, D. Geary, \& K. Koepke (Eds.), Language and Culture in Mathematical Cognition (pp. 197-227). Academic Press. https://doi.org/10.1016/B978-0-12-812574-8.00009-2

Sarnecka, B. W., \& Lee, M. D. (2009). Levels of number knowledge during early childhood. Journal of Experimental Child Psychology, 103(3), 325-337. https://doi.org/10.1016/j.jecp.2009.02.007

Schmittau, J. (2004). Vygotskian theory and mathematics education: Resolving the conceptual-procedural dichotomy. European Journal of Psychology of Education, 19(1), 19-43. https://doi.org/10.1007/ BF03173235

Schollar, E. (2015). The primary mathematics research project: 2004-2012. An evidence-based programme of research into understanding and improving the outcomes of mathematical education in South African primary schools. [Dissertation]. University of Cape Town: Department of Sociology.

Sinclair, N., \& Coles, A. (2017). Returning to ordinality in early number sense: Neurological, technological and pedagogical considerations. In E. Faggiano, F. Ferrara, \& A. Montone (Eds.), Innovation and technology enhancing mathematics education. Perspectives in the digital era (pp. 39-58). Springer Nature.

Steffe, L., Cobb, P., \& von Glasersfeld, E. (1988). Construction of arithmetical meanings and strategies. Springer Verlag.

Steffe, L. P. (2004). PSSM from a constructivist perspective. In D. H. Clements, J. Sarama, \& A.-M. DiBiase (Eds.), Engaging young children in mathematics: Standards for early childhood mathematics education. Lawrence Erlbaum.

Venkat, H., Askew, M., Watson, A., \& Mason, J. (2019). Architecture of mathematical structure. For the Learning of Mathematics, 39(1), 13-17.

Wynn, K. (1992). Children's acquisition of the number words and the counting system. Cognitive Psychology, 24(2), 220-251.

Wynn, K. (1998). Numerical competence in infants. In C. Donlan (Ed.), The development of mathematical skills (pp. 3-25). Psychology Press.

Publisher's note Springer Nature remains neutral with regard to jurisdictional claims in published maps and institutional affiliations. 